\title{
Entire Functions of Several Complex Variables Bounded Outside a Set of Finite Volume
}

\author{
By \\ Yasuichiro NisHIMURA*
}

Introduction

In this paper, we generalize two theorems of A. Edrei and P. Erdös [2] for the 1 dimensional case to the $n(\geqq 2)$ dimensional case.

The first one is the following:

Theorem 1. Let $f(z)$ be a nonconstant holomorphic function on $\mathbb{C}^{n}$ (i.e., entire function of $n$ complex variables) such that

$$
\text { (A) } \begin{aligned}
\liminf _{r \rightarrow+\infty} \frac{\log \log \log M(r)}{\log r} & <2 n \\
(M(r) & \left.=\max _{\|z\|=r}|f(z)|,\|z\|=\left(\left|z_{1}\right|^{2}+\cdots+\left|z_{n}\right|^{2}\right)^{1 / 2}\right) .
\end{aligned}
$$

Then, for every positive constant $B$, it satisfies the condition

(B) $m_{2 n}\left(\left\{z \in C^{n}|| f(z) \mid>B\right\}\right)=+\infty$,

where $m_{2 n}$ denotes the $2 n$ dimensional Lebesgue measure.

(Remark. The case $n=1$ is the Edrei-Erdös theorem.)

The second result of [2] is the construction of an example which shows that, when $n=1$, the constant $2 n=2$ in the right side of the inequality (A) is the largest possible in order to ensure the condition (B).

Let us recall this example. Let $\Omega$ be the domain in $\mathbb{C}$ defined by

$$
\text { ( } \Omega .1) \quad \Omega=\left\{w=x+i y \mid e^{2}<x,-\frac{\pi}{2 x(\log x)^{2}}<y<\frac{\pi}{2 x(\log x)^{2}}\right\} .
$$

Note that we have

$$
(\Omega .2) \quad m_{2}(\Omega)=\frac{\pi}{2}<+\infty
$$

Communicated by S. Nakano, October 7, 1986. Revised November 27, 1986.

* Department of Mathematics, Osaka Medical College, Takatsuki City 569, Osaka, Japan. 
where $m_{2}$ denotes the 2 dimensional Lebesgue measure. Then, the holomorphic function $\Phi(w)$ on $C$, which we call the Edrei-Erdös function in this paper, has the following two properties:

(E. 1) $\Phi(w)$ remains bounded for $w \in C-\Omega$,

(E. 2) $\Phi(w)-\exp \left(\exp (w \log w)^{2}\right)$ remains bounded for $w \in \Omega$, where the branch of $\log w$ is determined so as to take real values for real $w \in \Omega$.

Especially, combining ( $\Omega .1$ ), ( $\Omega .2$ ), (E.1) and (E.2), we have

(E. 3) $\lim _{r \rightarrow+\infty} \frac{\log \log \log M(r)}{\log r}=2 \quad\left(M(r)=\max _{|w|=r}|\Phi(w)|\right)$,

(E. 4) $\left\{w \in \boldsymbol{C}|| \Phi(w) \mid>B_{1}\right\} \subset \Omega$ and hence $m_{2}\left(\left\{w \in \boldsymbol{C}|| \Phi(w) \mid>B_{1}\right\}\right)<+\infty$ for some suitable $B_{1}>0$.

In order to state our second result, we need to introduce some special polynomials. For each integer $k \geqq 1$, we define the polynomials $Q_{n, k}(z)$ of $n$ variables $z=\left(z_{1}, \cdots, z_{n}\right)$ inductively on $n(\geqq 2)$ by

(Q. 1) $Q_{2, \dot{k}}\left(z_{1}, z_{2}\right)=z_{1}{ }^{2}+z_{2}{ }^{k}$ $Q_{n, k}\left(z_{1}, z_{2}, \cdots, z_{n}\right)=Q_{2, k}\left(z_{1}, Q_{n-1, k}\left(z_{2}, \cdots, z_{n}\right)\right)(n \geqq 3)$.

Observe the following properties:

(Q.2) degree of $Q_{n, k}(z)=k^{n-1}$,

(Q.3) $Q_{n, k}(0, \cdots, 0, r)=r^{k n-1}$.

The following theorem will be proved in $\S 2$.

Theorem 2. If $k \geqq 4$, we have

(QQ.1) $m_{2 n}\left(\left\{z \in \boldsymbol{C}^{n} \mid Q_{n, k}(z) \in \Omega\right\}\right)<+\infty$.

Consider the composition $\Phi_{n, k}=\Phi \cdot Q_{n, k}$ of the Edrei-Erdös function $\Phi$ and the polynomial $Q_{n, k}$. Then, combining (E.3), (E.4), (Q.2), (Q.3) and Theorem 2, we have

(Ф.1) $\lim _{r \rightarrow+\infty} \frac{\log \log \log M(r)}{\log r}=2 k^{n-1} \quad\left(M(r)=\max _{\|z\|=r}\left|\Phi_{n, k}(z)\right|\right)$,

(Ф.2) $\left\{z \in C^{n}|| \Phi_{n, k}(z) \mid>B_{1}\right\} \subset\left\{z \in C^{n} \mid Q_{n, k}(z) \in \Omega\right\}$, hence $m_{2 n}\left(\left\{z \in C^{n}|| \Phi_{n, k}(z) \mid>B_{1}\right\}\right)<+\infty$ when $k \geqq 4$, where $B_{1}$ is the constant in (E.4).

Especially, when $k=4$, we have

Corollary. The holomorphic function $\Phi_{n, 4}(z)$ on $C^{n}(n \geqq 2)$ has the following two properties:

(C) $\lim _{r \rightarrow+\infty} \frac{\log \log \log M(r)}{\log r}=2^{2 n-1} \quad\left(M(r)=\max _{\|z\|=r}\left|\Phi_{n, 4}(z)\right|\right)$,

(D) $m_{2 n}\left(\left\{z \in C^{n} ;\left|\Phi_{n, 4}(z)\right|>B_{1}\right\}\right)<+\infty$, where $B_{1}$ is the constant in (E. 4). 
Remark. Compare this Corollary with Theorem 1. Then, because of the difference between $2 n$ in (A) and $2^{3 n-1}$ in (C), our results leave something to be improved.

Such problems as are dealt with in this paper were also treated by A. A. Gol'dberg [4] and L. J. Hansen [5] for the case $n=1$. Moreover, G. A. Camera [1] considered the case of subharmonic functions in $R^{m}$ (see Remark to Theorem 1 in $\S 1$ ).

In $\S 1$, we prove Theorem 1 . For that purpose, for each point $x$ in the unit sphere $S=\{z \mid\|z\|=1\}$ in $\mathbb{C}^{n}$, we consider the so-called slice function $f_{x}(t)=f(t x)$ which is a holomorphic function in $t \in C$. We adapt the argument of [2] for these slice functions $\left\{f_{x} \mid x \in S\right\}$.

In $\S 2$, after making some observations about the polynomials $\left\{Q_{n}{ }_{k}(z)\right\}$, we prove Theorem 2 .

Acknowledgement. The referee made some useful comments. Especially, the simple proof of Lemma 4 in $\S 2$ is due to him. The author thanks him for these things.

\section{$\S 1$. Proof of Theorem $\mathbb{1}$}

In [2], Edrei and Erdös used a lemma of Borel type to prove Theorem 1 when $n=1$. In our proof of Theorem 1 when $n \geqq 2$, we use a lemma of the same type. So we prepare it first of all.

Lemma 1. Let $U(r)$ be a positive continuous nondecreasing function on $[1, \infty)$ such that

$$
U(1)>e \text {. }
$$

Let $\delta>0$ be an arbitrarily chosen constant. Then, there exists a closed subset $\varepsilon \subset[1, \infty)$ such that

(a) for every $r \notin \mathcal{E}$ with $r \geqq 1$,

$$
U\left(r+r(\log U(r))^{-(1+o)}\right)<e U(r) ;
$$

(b) for every $s, t$ with $1 \leqq s<t<\infty$ aind with

$$
\delta(\log U(s)-1)^{\hat{o}}>4
$$

we have

$$
m_{1}(\mathcal{E}(s, t))<\frac{t}{4}
$$

where $\mathcal{E}(s, t)=\{r \mid s \leqq r \leqq t, r \in \mathcal{E}\}$ and $m_{\text {. donotes }}$ the 1 dimcrisional Lebesgue measure. 
Remark. A sharper form of Lemma 1 is found in the paper of Edrei and Fuchs [3]. But in their statement, the extent of $s$ (the above condition (1.3)) to ensure the estimate (1.4) is implicit. As we need (1.3) later on, we give the proof of Lemma 1.

Proof. Put $\phi(x)=\log U\left(e^{x}\right) \quad(0 \leqq x<\infty)$. Since $U(r)$ is nondecreasing, (1.1) implies $\phi(x)>1 \quad(0 \leqq x<\infty)$. Consider the function $h(x)=x^{-(1+\delta)}(0<x<\infty)$, and put $H(x)=\phi(x+h(\phi(x)))-\phi(x)-1 \quad(0 \leqq x<\infty)$. Then $H(x)$ is well-defined and continuous. Put $E=\{x \mid 0 \leqq x, H(x) \geqq 0\}$ and $\mathcal{E}=\left\{r \mid r=e^{x}, x \in E\right\}$. Then $E$ and $\mathcal{E}$ are closed subsets of $[0, \infty)$ and $[1, \infty)$ respectively. Using the inequality $e^{\alpha} \geqq \alpha+1$, we can see that (a) holds.

In order to prove (b), put $y=\log s, z=\log t$ and $E(y, z)=E \cap[y, z]$. We claim

$$
m_{1}(E(y, z)) \leqq \int_{\phi(y)-1}^{\phi(z)} h(x) d x
$$

In fact, we define $y_{1}, y_{1}^{\prime}, y_{2}, y_{2}^{\prime}, \cdots$ inductively by

$$
\begin{array}{ll}
y_{1}=\min E(y, z) & y_{1}^{\prime}=y_{1}+h\left(\phi\left(y_{1}\right)\right) \\
y_{n}=\min E\left(y_{n-1}^{\prime}, z\right) & y_{n}^{\prime}=y_{n}+h\left(\phi\left(y_{n}\right)\right) \quad(n \geqq 2)
\end{array}
$$

where $E\left(y_{n-1}^{\prime}, z\right)=E \cap\left[y_{n-1}^{\prime}, z\right]$. Since $\phi\left(y_{n}^{\prime}\right)-\phi\left(y_{n}\right) \geqq 1$, there exists some integer $N \geqq 1$ such that $y_{N}^{\prime}>z$ or $y_{N}^{\prime} \leqq z$ with $E\left(y_{N}^{\prime}, z\right)=\varnothing$. Hence $E(y, z) \subset \bigcup_{n=1}^{N}\left[y_{n}, y_{n}^{\prime}\right]$. Noting that $\phi\left(y_{n}\right)-\phi\left(y_{n-1}\right) \geqq 1(n \geqq 2)$, we have

$$
\begin{aligned}
m_{1}(E(y, z)) \leqq & \sum_{n=1}^{N}\left(y_{n}^{\prime}-y_{n}\right) \\
& =\sum_{n=1}^{N} h\left(\phi\left(y_{n}\right)\right) \\
& \leqq h\left(\phi\left(y_{1}\right)\right)+\sum_{n=2}^{N} h\left(\phi\left(y_{n}\right)\right)\left(\phi\left(y_{n}\right)-\phi\left(y_{n-1}\right)\right) \\
& \leqq h\left(\phi\left(y_{1}\right)\right)+\int_{\phi\left(y_{1}\right)}^{\phi\left(y_{N}\right)} h(x) d x \\
& \leqq \int_{\phi\left(y_{1}\right)-1}^{\phi(z)} h(x) d x \\
& \leqq \int_{\phi(y)-1}^{\phi(z)} h(x) d x
\end{aligned}
$$

Then, in view of (1.3), a calculation yields

$$
\begin{aligned}
m_{1}(\mathcal{E}(s, t)) & \leqq t \delta^{-1}(\log U(s)-1)^{-\delta} \\
& \leqq \frac{t}{4}
\end{aligned}
$$

This proves Lemma 1. 
Let $\boldsymbol{C}^{n}$ be the $n$ dimensional complex Euclidean space, and let $S$ be the unit sphere $\{z \mid\|z\|=1\}$ in $C^{n}$. Especially, the unit circle (when $n=1$ ) is denoted by $T: T=\left\{e^{i \theta} \mid 0 \leqq \theta \leqq 2 \pi\right\}$. We denote by $d m_{2 n}$ the $2 n$ dimensional Lebesgue measure on $C^{n}$, by $d S$ the rotation-invariant area element of $S$ and by $d \theta$ the line element of $T$. Considering the identification $\mathbb{C}^{n}-\{0\}=(0, \infty) \times S$, we obtain $d m_{2 n}=r^{2 n-1} d r d S$.

Definition 1. Let $f(z)$ be a holomorphic function on $\mathbb{C}^{n}(n \geqq 2)$. For $r>0$ and $x \in S$, define

$$
\begin{aligned}
& M_{f}(r, x)=\max _{e^{2 \theta} \in T}\left|f\left(r e^{i \theta} x\right)\right| \\
& T_{f}(r, x)=\frac{1}{2 \pi} \int_{T} \log ^{+}\left|f\left(r e^{i \theta} x\right)\right| d \theta
\end{aligned}
$$

where $\log ^{+} t=\max (\log t, 0)(t>0)$. Then these two functions are continuous in $(0, \infty) \times S$.

Concerning the functions $M_{f}(r, x)$ and $T_{f}(r, x)$, the following facts are well known.

Lemma 2. Let $f(z)$ be a holomorphic function on $\mathbb{C}^{n}(n \geqq 2)$. Then $M_{f}(r, x)$ and $T_{f}(r, x)$ are related by the following inequalities:

$$
T_{f}(r, x) \leqq \log ^{+} M_{f}(r, x) \leqq \frac{s+r}{s-r} T_{f}(s, x) \quad(0<r<s) .
$$

For $x \in S$ such that the slice function $f_{x}(t)=f(t x)(t \in C)$ is not a constant function, $M_{f}(r, x)$ and $T_{f}(r, x)$ are unbounded continuous increasing function in $r$.

Definition 2. Let $f(z)$ be a holomorphic function on $\mathbb{C}^{n}(n \geqq 2)$. For $r>0$ and $x \in S$, we put

$$
\begin{aligned}
& \Lambda_{f}(r, x)=\left\{e^{i \theta} \in T|\log | f\left(r e^{i \theta} x\right) \mid>\frac{1}{2} T_{f}(r, x)\right\} \\
& \Lambda_{f}(r)=\left\{y \in S|\log | f(r y) \mid>\frac{1}{2} T_{f}(r, y)\right\} \\
& \Lambda_{f}=\left\{z \in C^{n}-\{0\}|\log | f(z) \mid>\frac{1}{2} T_{f}\left(\|z\|,\|z\|^{-1} z\right)\right\} .
\end{aligned}
$$

Then $\Lambda_{f}(r, x), \Lambda_{f}(r)$ and $\Lambda_{f}$ are open subsets of $T, S$ and $C^{n}-\{0\}$ respectively.

A subset $M \subset S$ is said to be circular if $e^{i \theta} x \in M$ whenever $x \in M$ and $e^{i \theta} \in T$.

Lemma 3. Let $f(z)$ be a holomorphic function on $\mathbb{C}^{n}(n \geqq 2)$. We put $l_{f}(r, x)=\int_{\Lambda_{f}(r, x)} d \theta$ on $(0, \infty) \times S$. Let $M$ be a measurable circular subset of $S$. 
(a) $l_{f}(r, x)$ is a measurable function in $(0, \infty) \times S$.

(b) For $r>0, \int_{M \cap \Lambda_{f}(r)} d S(x)=\frac{1}{2 \pi} \int_{M} l_{f}(r, x) d S(x)$.

Proof. Consider the characteristic function $g(z)$ of the open subset $\Lambda_{f}$ of $C^{n}-\{0\}$ (i.e., $g(z)=0$ for $z \in C^{n}-\left(\{0\} \cup \Lambda_{f}\right) ; g(z)=1$ for $\left.z \in \Lambda_{f}\right)$. Consider the mapping $\tau:(0, \infty) \times S \times T \rightarrow C^{n}-\{0\}$ defined by $\tau\left(r, x, e^{\imath \theta}\right)=r e^{i \theta} x$. Then we have

$$
l_{f}(r, x)=\int_{T} g \circ \tau\left(r, x, e^{\imath \theta}\right) d \theta
$$

So, from Fubini's theorem applied to the function $g \circ \tau\left(r, x, e^{2 \theta}\right)$ on $(0, \infty) \times S \times T$, the assertion (a) follows.

Next, consider the characteristic function $h(x)$ of the subset $\Lambda_{f}(r)$ of $S$, and the mapping $\rho: S \times T \rightarrow S$ defined by $\rho\left(x, e^{i \theta}\right)=e^{2 \theta} x$. Then according to Fubini's theorem, we have

$$
\begin{aligned}
& \int_{M \rtimes T} h \circ \rho\left(x, e^{i \theta}\right) d S(x) d \theta \\
= & \int_{T} d \theta \int_{M} h \circ \rho\left(x, e^{i \theta}\right) d S(x) .
\end{aligned}
$$

Since $d S$ is rotation-invariant and $M$ is circular, the inner integral in the last integral is independent of $e^{i \theta}$ and is equal to $\int_{M_{\cap} \Lambda_{f}(r)} d S(x)$. On the other hand,

$$
\begin{aligned}
& \int_{M \times T} h \circ \rho\left(x, e^{i \theta}\right) d S(x) d \theta \\
= & \int_{M} d S(x) \int_{T} h \circ \rho\left(x, e^{2 \theta}\right) d \theta \\
= & \int_{M} l_{f}(r, x) d S(x) .
\end{aligned}
$$

Consequently, the assertion (b) is proved.

Proof of Theorem 1 when $n \geqq 2$. Let $f(z)$ be a nonconstant holomorphic function on $\mathbb{C}^{n}$ satisfying the condition (A). We fix a positive constant $B$. For simplicity, we assume

$$
|f(0)|>\exp (e) .
$$

Note that this assumption is always fulfilled if we replace $f(z)$ by $f\left(z-z_{0}\right)$ for a suitable $z_{0} \in C^{n}$, and that this replacement does not change the assumption (A) nor the conclusion (B).

According to the assumption (A), there exist a corstant $\eta>0$ and an infinite set $I$ of positive numbers with $\sup \{r \mid r \in I\}=+\infty$ such that 


$$
\log \log M(r)<r^{2 n(1-\eta)} \quad \text { for every } r \in I .
$$

We take $a>0$ which is big enough to yield

$$
\eta(a-1)^{\eta}>4
$$

Next, define the subset $Q \subset S$ by

$$
Q=\left\{x \in S \mid T_{f}(r, x) \text { is not bounded as } r \rightarrow \infty\right\} .
$$

Since $S-Q=\left\{x \in S \mid f_{x}(t)\right.$ is identically equal to $\left.f(0)\right\}, S-Q$ is a closed subset of measure 0 . So $Q$ is an open subset, and putting $c_{n}=\int_{S} d S$, we have

$$
\int_{Q} d S=c_{n}
$$

For $r>0$, let $Q(r)$ be the open subset of $Q$ defined by

$$
Q(r)=\left\{x \in Q \mid T_{f}(r, x)>\max \left(e^{a}, 2 \log B\right)\right\} .
$$

Then from $Q(r) \subset Q^{\prime}\left(r^{\prime}\right)\left(r<r^{\prime}\right)$ and $Q=\bigcup_{r>0} Q(r)$, (1.9) yields

$$
\lim _{r \rightarrow+\infty} \int_{Q(r)} d S=c_{n}
$$

Hence we can choose a constant $r_{1}>0$ such that

$$
\int_{Q\left(r_{1}\right)} d S \geqq \frac{1}{2} c_{n} .
$$

For $R \geqq r_{1}$, we define the open subset $G(R)$ of $\mathbb{C}^{n}$ by

$$
G(R)=\left\{z \in \mathbb{C}^{n}|R<\|z\|<2 R, \log | f(z) \mid>\frac{1}{2} T_{f}\left(\|z\|,\|z\|^{-1} z\right),\|z\|^{-1} z \in Q\left(r_{1}\right)\right\} .
$$

Then for every $z \in G(R)$, in view of (1.10), we have

$$
\log |f(z)|>\frac{1}{2} T_{f}\left(\|z\|,\|z\|^{-1} z\right)>\frac{1}{2} T_{f}\left(r_{1},\|z\|^{-1} z\right)>\log B
$$

which implies

$$
\left\{z \in \mathbb{C}^{n}|| f(z) \mid>B\right\} \supset G(R) .
$$

In order to prove Theorem 1, we shall estimate from below the measure $m_{2 n}(G(R))$. We start with the following obvious inequality:

$$
T_{f}(r, x) \leqq \frac{1}{2 \pi} \int_{\Lambda_{f}(r, x)} \log M_{f}(r, x) d \theta+\frac{1}{2 \pi} \int_{T} \frac{1}{2} T_{f}(r, x) d \theta .
$$

From this inequality, we have 


$$
l_{f}(r, x) \geqq \frac{\pi T_{f}(r, x)}{\log M_{f}(r, x)}
$$

In order to estimate the right side of (1.13), we apply Lemma 1. In view of (1.6), $U(r)=T_{f}(r, x)(x \in S)$ satisfies (1.1). Take the number $\eta$ in (1.7) as $\delta$ in Lemma 1. Note that, for $x \in Q\left(r_{1}\right),(1.8)$ and (1.10) implies $\eta\left(\log T_{f}\left(r_{1}, x\right)-1\right)^{\eta}$ $>4$, which corresponds to the condition (1.3). Hence for each $x \in Q\left(r_{1}\right)$, there exists a closed subset $\mathcal{E}(x) \subset[1, \infty)$ such that

(a) for every $r \notin \mathcal{E}(x)$ with $r \geqq 1$,

$$
T_{f}\left(r+r\left(\log T_{f}(r, x)\right)^{-(1+\eta)}\right)<e T_{f}(r, x),
$$

(b) for every $R \geqq r_{1}$

$$
m_{1}(\mathcal{E}(R, 2 R ; x))<\frac{R}{2},
$$

where $\mathcal{E}(R, 2 R ; x)=\{r \mid R \leqq r \leqq 2 R, r \in \mathcal{E}(x)\}$.

Especially, for $r \geqq 1$ with $r \notin \mathcal{E}(x)$, (1.5) and (1.14) yield

$$
\log M_{f}(r, x) \leqq 3 e\left(\log T_{f}(r, x)\right)^{1+\eta} T_{f}(r, x)
$$

and hence, in view of (1.13)

$$
l_{f}(r, x) \geqq e^{-1}\left(\log T_{f}(r, x)\right)^{-(1+\eta)} .
$$

Note that $Q\left(r_{1}\right)$ is a circular subset of $S$. Hence we can apply Lemma 3. According to Fubini's theorem, (1.15) and (1.16), we have

$$
\begin{aligned}
m_{2 n}(G(R)) & =\int_{R}^{2 R} r^{2 n-1} d r \int_{Q\left(r_{1}\right) \cap A_{f}(r)} d S(x) \\
& =\int_{R}^{2 R} r^{2 n-1} d r \frac{1}{2 \pi} \int_{Q\left(r_{1}\right)} l_{f}(r, x) d S(x) \\
& \geqq R^{2 n-1} \int_{Q\left(r_{1}\right)} d S(x) \frac{1}{2 \pi} \int_{[R, 2 R]-\varepsilon(R, 2 R ; x)} l_{f}(r, x) d r \\
& \geqq \frac{1}{4 \pi e} R^{2 n} \int_{Q\left(r_{1}\right)}\left(\log T_{f}(2 R, x)\right)^{-(1+\eta)} d S(x) .
\end{aligned}
$$

If we choose $R$ such that $2 R \in I$, then (1.5), (1.7) and (1.11) yield

$$
m_{2 n}(G(R)) \geqq \frac{c_{n}}{8 \pi e} R^{2 n}(2 R)^{-2 n\left(1-\eta^{2}\right)}=\tilde{c}_{n} R^{2 n \eta^{2}} .
$$

Hence letting $R \rightarrow+\infty$ with $2 R \in I$, we find that $\limsup _{R \rightarrow+\infty} m_{2 n}(G(R))=+\infty$. In view of (1.12) we conclude (B). 
Remark. Let $u(x)$ be a subharmonic function in $\mathbb{R}^{n}(m \geqq 2)$. We put $B(r)$ $=\max _{\|x\|=r} u^{+}(x)$ and $T(r)=\frac{1}{c_{m}} \int_{x \in S} u^{+}(r x) d S(x)$, where $d S$ is the area element of $S=\{\|x\|=1\}, \quad c_{m}=\int_{x \in S} d S(x)$ and $u^{+}=\max (u, 0)$. Then they are related as follows :

$$
T(r) \leqq B(r) \leqq \frac{s^{m-2}(s+r)}{(s-r)^{m-1}} T(s) \quad(0<r<s) .
$$

By a direct adaptation of the argument of [2], using the above inequalities, we can prove the following:

If $u(x)$ is not bounded above and satisfies

$$
\liminf _{r \rightarrow+\infty} \frac{\log \log B(r)}{\log r}<\frac{m}{m-1},
$$

then, for every real constant $B$,

$$
m_{m}\left(\left\{x \in \mathbb{R}^{m} \mid u(x)>B\right\}\right)=+\infty .
$$

As was shown in [1], the constant $m(m-1)^{-1}$ on the right side of (1.17) is the largest possible. On the other hand, the constant on the right side of $(A)$ in Theorem 1 is not $2 n(2 n-1)^{-1}$ but $2 n$. This improvement seems to come from the fact that slice function $\varphi_{x}(t)=\varphi(t x)(x \in S, t \in \mathbb{C})$ of a plurisubharmonic function $\varphi$ in $\mathbb{C}^{n}$ is subharmonic (or $\equiv-\infty$ ), while the restriction to a proper linear subspace of a subharmonic function in $\mathbb{R}^{m}$ is not necessarily subharmonic.

\section{§. Proof of Theorem 2}

Recall the polynomials $Q_{n, k}(z)$ which were introduced in Introduction. We shall make some preparations in order to prove the property ( $2 \mathrm{Q} .1$ ) (Theorem 2 ).

For integers $n \geqq 2, k \geqq 1$ and $N \geqq 1$, we put

$$
J_{n, k}(N)=m_{2 n}\left(\left\{z \in C^{n}|N-1 \leqq| Q_{n, k}(z) \mid \leqq N\right\}\right) .
$$

We shall estimate $J_{n, k}(N)$.

First of all, we confine ourselves to the case $n=2$. For integers $k \geqq 1$ and $N \geqq 1$, and for $z_{2} \in C$, we pose

$$
\begin{aligned}
s_{k}\left(z_{2}, N\right) & =m_{2}\left(\left\{z_{1} \in C|N-1 \leqq| Q_{2, k}\left(z_{1}, z_{2}\right) \mid \leqq N\right\}\right) \\
& =m_{2}\left(\left\{z_{1} \in \mathbb{C}|N-1 \leqq| z_{1}{ }^{2}+z_{2}{ }^{k} \mid \leqq N\right\}\right) .
\end{aligned}
$$

Put $r=\left|z_{2}\right|$. Then $s_{k}\left(z_{2}, N\right)$ depends only on $r$ :

$$
s_{k}\left(z_{2}, N\right)=s_{k}(r, N)=m_{2}\left(\left\{z_{1} \in C|N-1 \leqq| z_{1}{ }^{2}+r^{k} \mid \leqq N\right\}\right) .
$$

Lemma 4. For $k \geqq 1$ and $N \geqq 1$, we have 


$$
\begin{array}{ll}
s_{k}(r, N) \leqq \alpha_{1} N^{1 / 2} & \text { if } r^{k} \leqq N \\
s_{k}(r, N) \leqq \alpha_{2} N^{3 / 2} r^{-k} & \text { if } r^{k} \geqq N
\end{array}
$$

where $\alpha_{1}$ and $\alpha_{2}$ are absolute constants.

Proof. Changing the notations for simplicity, we put

$$
D=\left\{z \in \mathcal{C}|\operatorname{Re} z>0, a<| z^{2}-c \mid<b\right\} \quad(0 \leqq a<b, c \geqq 0) .
$$

We estimate the area of the domain $D$. Consider the holomorphic function $w=z^{2}$ and the annulus

$$
D^{\prime}=\{w \in C|a<| w-c \mid<b\} .
$$

Then by this function, $D$ is mapped conformally onto the domain $D^{\prime}-\{$ the negative part of the real axis\}. Consequently, taking the polar coordinates $w=\rho e^{i \phi}$,

$$
\begin{aligned}
m_{2}(D) & =\int_{D} d m_{2}(z) \\
& =\int_{D^{\prime}}\left|\frac{d z}{d w}\right|^{2} d m_{2}(w) \\
& =\int_{D^{\prime}} \frac{1}{4|w|} d m_{2}(w) \\
& =\frac{1}{4} \int_{D^{\prime}} d \rho d \phi .
\end{aligned}
$$

Denote by $l(\phi)$ the length of the intersection of the half line $\{\arg w=\phi\}$ with $D^{\prime}(0 \leqq \phi \leqq 2 \pi)$. Then, we have

$$
m_{2}(D)=\frac{1}{4} \int_{0}^{2 \pi} l(\phi) d \phi
$$

We estimate $l(\phi)$ by examining the two cases below separately.

(i) When $b \geqq c$, we have $l(\phi)+l(\phi+\pi) \leqq 2\left(b^{2}-a^{2}\right)^{1 / 2} \quad(0 \leqq \phi \leqq \pi)$. Hence, $m_{2}(D) \leqq \frac{\pi}{2}\left(b^{2}-a^{2}\right)^{1 / 2}$.

(ii) When $b \leqq c$, we have $l(\phi)>0$ only when $|\phi|<\operatorname{Arc} \sin \frac{b}{c}$. We also have $l(\phi) \leqq 2\left(b^{2}-a^{2}\right)^{1 / 2}$. It follows that

$$
m_{2}(D) \leqq\left(b^{2}-a^{2}\right)^{1 / 2} \operatorname{Arc} \sin \frac{b}{c} \leqq \frac{\pi}{2}\left(b^{2}-a^{2}\right)^{1 / 2} \frac{b}{c} .
$$

Thus, putting $a=N-1$ and $b=N$, we obtain (2.1).

Lemma 5. If $k \geqq 3$, then for every $N \geqq 1$, we have

(Q. 4) $\quad J_{2, k}(N) \leqq c N^{(k+4) / 2 k}$ 
where $c>0$ is some absolute constant.

Proof. For a positive integer $M$, we put

$$
S_{k}(M, N)=\max _{M-1 \leqq r \leqq M} s_{k}(r, N) .
$$

Then, we have

$$
J_{2, k}(N) \leqq 2 \pi \sum_{M=1}^{\infty} M S_{k}(M, N) .
$$

It can be easily deduced from Lemma 4 that

$$
\begin{array}{ll}
S_{k}(M, N) \leqq \beta_{1} N^{1 / 2} & \text { if } M \leqq N^{1 / k}+2 \\
S_{k}(M, N) \leqq \beta_{2} N^{3 / 2}(M-1)^{-k} & \text { if } M \geqq N^{1 / k}+2
\end{array}
$$

where $\beta_{1}=\max \left(\alpha_{1}, \alpha_{2}\right)$ and $\beta_{2}=\alpha_{2}$.

When $k \geqq 3$, according to (2.3) and the following estimates

$$
\begin{aligned}
& \sum M \leqq\left(N^{1 / k}+2\right)^{2} \leqq 9 N^{2 / k} \\
& \sum M(M-1)^{-k} \leqq \int_{N^{1 / k}+1}^{\infty} x(x-1)^{-k} d x \leqq 2 N^{(-k+2) / k}
\end{aligned}
$$

where the first and the second summations extend over integers with $1 \leqq M \leqq N^{1 / k}+2$ and $N^{1 / k}+2 \leqq M<+\infty$ respectively, we obtain

$$
2 \pi \sum_{M=1}^{\infty} M S_{k}(M, N) \leqq c N^{(k+4) / 2 k}
$$

in which we put $c=2\left(9 \beta_{1}+2 \beta_{2}\right)$. In view of (2.2) and (2.4), Lemma 5 is proved.

Now, we return to the general case $n \geqq 2$.

Lemma 6. Let $n \geqq 2$ and $N \geqq 1$ be integers. If $k \geqq 4$,

(Q.4) $\quad J_{n, k}(N) \leqq c^{n-1} N^{(k+4) / 2 k}$

where $c>0$ is the absolute constant in Lemma 5.

Proof. We shall proceed by induction on $n$. The case $n=2$ was proved in Lemma 5. Assume that $n \geqq 3$. Then observing

$$
\begin{aligned}
& m_{n_{n}}\left(\left\{z \in C^{n}|N-1 \leqq| z_{1}{ }^{2}+Q_{n-1, k}\left(z_{2}, \cdots, z_{n}\right)^{k} \mid \leqq N,\right.\right. \\
& \left.\left.\quad M-1 \leqq\left|Q_{n-1, k}\left(z_{2}, \cdots, z_{n}\right)\right| \leqq M\right\}\right) \\
& \leqq m_{2 n-2}\left(\left\{\left(z_{2}, \cdots, z_{n}\right) \in \mathbb{C}^{n-1}|M-1 \leqq| Q_{n-1, k}\left(z_{2}, \cdots, z_{n}\right) \mid \leqq M\right\}\right) S(M, N) \\
& =J_{n-1, k}(M) S_{k}(M, N),
\end{aligned}
$$

we obtain 


$$
J_{n, k}(N) \leqq \sum_{M=1}^{\infty} J_{n-1, k}(M) S_{k}(M, N)
$$

According to the induction hypothesis, (2.4) and (2.5), when $k \geqq 4$,

$$
\begin{aligned}
J_{n, k}(N) & \leqq c^{n-2} \sum_{M=1}^{\infty} M^{(k+4) / 2 k} S_{k}(M, N) \\
& \leqq c^{n-2} 2 \pi \sum_{M=1}^{\infty} M S_{k}(M, N) \\
& \leqq c^{n-1} N^{(k+4) / 2 k}
\end{aligned}
$$

Thus, Lemma 6 is proved.

For $e^{i \theta} \in T$, let $R_{\theta}: C \rightarrow C$ be the rotation defined by $R_{\theta}(w)=e^{i \theta} u$. For a subset $E \subset C$, the image of $E$ under $R_{\theta}$ will be denoted by $R_{\theta}(E)$.

Lemma 7. For $e^{2 \theta} \in T$, we have

(Q.5) $\quad m_{2 n}\left(\left\{z \mid Q_{n, k}(z) \in E\right\}\right)=m_{2 n}\left(\left\{z \mid Q_{n, k}(z) \in R_{\theta}(E)\right\}\right)$

where both sides may be infinite simultaneously.

Proof. For simplicity, we shall prove Lemma 7 only when $n=2$, writing $Q\left(z_{1}, z_{2}\right)$ in place of $Q_{2, k}\left(z_{1}, z_{2}\right)$. The proof for the general case is similar. Define a unitary transformation $U$ of $C^{2}$ by

$$
U\left(z_{1}, z_{2}\right)=\left(\exp \left(-\frac{i \theta}{2}\right) z_{1}, \exp \left(-\frac{i \theta}{k}\right) z_{2}\right) .
$$

Then we have $Q \cdot U\left(z_{1}, z_{2}\right)=e^{-i \theta} Q\left(z_{1}, z_{2}\right)$. The invariance of the Lebesgue measure under the unitary transformations yields

$$
\begin{aligned}
& m_{4}\left(\left\{\left(z_{1}, z_{2}\right) \mid Q\left(z_{1}, z_{2}\right) \in R_{\theta}(E)\right\}\right) \\
= & m_{4}\left(\left\{\left(z_{1}, z_{2}\right) \mid Q \cdot U\left(z_{1}, z_{2}\right) \in E\right\}\right) \\
= & m_{4}\left(\left\{\left(z_{1}, z_{2}\right) \mid Q\left(z_{1}, z_{2}\right) \in E\right\}\right) .
\end{aligned}
$$

In addition, we prepare the following lemma concerning the domain $\Omega$.

Lemma 8. There exists an absolute constant $\beta$ such that, for every integer $N>e^{2}$,

$$
\begin{array}{r}
\{w \in C|N-1 \leqq| w \mid \leqq N, w \in \Omega\} \subset\{w \in C|N-1 \leqq| w \mid \leqq N, \\
\left.|\arg (w)| \leqq \beta(N \log N)^{-2}\right\} .
\end{array}
$$

Proof is quite obvious.

Now, we can prove Theorem 2 . 
Proof. According to Lemma 7 and Lemma 8,

$$
\begin{aligned}
& m_{2 n}\left(\left\{z \in \mathbb{C}^{n}|N-1 \leqq| Q_{n, k}(z) \mid \leqq N, Q_{n, k}(z) \in \Omega\right\}\right) \\
\leqq & \pi^{-1} \beta(N \log N)^{-2} J_{n, k}(N) \quad\left(N>e^{2}\right) .
\end{aligned}
$$

Consequently, when $k \geqq 4$, Lemma 6 yields

$$
\begin{aligned}
& m_{2 n}\left(\left\{z \in \mathbb{C}^{n} \mid Q_{n, k}(z) \in \Omega\right\}\right) \\
\leqq & \pi^{-1} \beta c^{n-1} \sum_{N=2}^{\infty} N^{(-3 k+4) / 2 k}(\log N)^{-2} \\
\leqq & \pi^{-1} \beta c^{n-1} \sum_{N=2}^{\infty} N^{-1}(\log N)^{-2}<+\infty
\end{aligned}
$$

where we used the fact that the measure on the left in (2.6) is equal to 0 when $N<e^{2}$. This proves Theorem 2 .

\section{References}

[1] Camera, G. A., Subharmonic functions on sets of finite measure, Quart. J. Math. Oxford Ser. (2), 33 (1982), 27-43.

[2] Edrei, A. and Erdös, P., Entire functions bounded outside a finite area, Acta Math. Hung., 45 (1985), 367-376.

[3] Edrei, A. and Fuchs, W.H. J., Bounds for the number of deficient values of certain classes of meromorphic functions, Proc. London Math. Soc., 12 (1962), 315-344.

[4] Gol'dberg, A. A., Sets on which the modulus of an entire function is bounded below, Siberian Math. J., 20 (1980), 360-364.

[5] Hansen, L.J., On the growth of entire functions bounded on large sets, Canad. J. Math., 29 (1977), 1287-1291. 
